Draft Version February 10, 2021

Typeset using LATEX twocolumn style in AASTeX63

\title{
An oversized magnetic sheath wrapping around the parsec-scale jet in $3 \mathrm{C} 273$
}

\author{
M. M. Lisakov (D) ${ }^{1,2}$ E. V. Kravchenko (D) ${ }^{3,2,4}$ A. B. Pushkarev (D) ${ }^{5,2,3}$ Y. Y. Kovalev (D), ${ }^{2,3,1}$ \\ T. K. Savolainen (iD, $6,7,1$ and M. L. Lister (iD) 8 \\ ${ }^{1}$ Max-Planck-Institut für Radioastronomie, Auf dem Hügel 69, Bonn 53121, Germany \\ ${ }^{2}$ Astro Space Center, Lebedev Physical Institute, Russian Academy of Sciences, Profsoyuznaya st., 84/32, Moscow, 117997, Russia \\ ${ }^{3}$ Moscow Institute of Physics and Technology, Institutsky per. 9, Moscow region, Dolgoprudny, 141700, Russia \\ ${ }^{4}$ INAF Istituto di Radioastronomia, Via P. Gobetti, 101, Bologna, 40129, Italy \\ ${ }^{5}$ Crimean Astrophysical Observatory, Nauchny 298688, Crimea, Russia \\ ${ }^{6}$ Aalto University Department of Electronics and Nanoengineering, PL 15500, FI-00076 Aalto, Finland \\ ${ }^{7}$ Aalto University Metsähovi Radio Observatory, Metsähovintie 114, FI-02540 Kylmälä, Finland \\ ${ }^{8}$ Department of Physics and Astronomy, Purdue University, 525 Northwestern Avenue, West Lafayette, IN 47907, USA
}

(Received 2 December 2020; Revised 27 January 2021; Accepted 29 January 2021)

Accepted to ApJ

\begin{abstract}
In recent studies, several AGN have exhibited gradients of the Faraday Rotation Measure (RM) transverse to their parsec-scale jet direction. Faraday rotation likely occurs as a result of a magnetized sheath wrapped around the jet. In the case of 3C 273, using Very Long Baseline Array multi-epoch observations at 5, 8 and $15 \mathrm{GHz}$ in 2009-2010, we observe that the jet RM has changed significantly towards negative values compared with that previously observed. These changes could be explained by a swing of the parsec-scale jet direction which causes synchrotron emission to pass through different portions of the Faraday screen. We develop a model for the jet-sheath system in $3 \mathrm{C} 273$ where the sheath is wider than the single-epoch narrow relativistic jet. We present our oversized sheath model together with a derived wide jet full intrinsic opening angle $\alpha_{\mathrm{int}}=2.1^{\circ}$ and magnetic field strength $B_{\|}=3 \mu \mathrm{G}$ and thermal particle density $N_{\mathrm{e}}=125 \mathrm{~cm}^{-3}$ at the wide jet-sheath boundary $230 \mathrm{pc}$ downstream (deprojected) from its beginning. Most of the Faraday rotation occurs within the innermost layers of the sheath. The model brings together the jet direction swing and long-term RM evolution and may be applicable to other AGN jets that exhibit changes of their apparent jet direction.
\end{abstract}

Keywords: galaxies: active - galaxies: radio jets - galaxies: quasars: individual (3C 273) - techniques: interferometric -

\section{INTRODUCTION}

General relativistic magnetohydrodynamic (GRMHD) simulations illustrate the key role of magnetic fields in the formation of relativistic jets in Active Galactic Nuclei (AGN; e.g. Meier et al. 2001; Vlahakis \& Königl 2004; Tchekhovskoy et al. 2011; Zamaninasab et al. 2014). The topology and structure of these magnetic fields remains an active area of research. The rotation of the central supermassive black hole twists

Corresponding author: Mikhail Lisakov

mlisakov@mpifr-bonn.mpg.de magnetic field lines into a helical shape that can be revealed by observations of transverse Faraday rotation gradients. GRMHD simulations also show that accreting supermassive black hole systems naturally form a spine-sheath structure of the relativistic jets as a result of transverse velocity stratification in the outflows (e.g. McKinney 2006; Nakamura et al. 2018). The sheath, which may be a magnetized screen surrounding the jet or the jet boundary layer, has been suggested as a plausible source of the Faraday rotation (e.g. Asada et al. 2008).

Very Long Baseline Interferometry (VLBI) observations of parsec-scale AGN jets (e.g., Zavala \& Taylor 2003, 2004; O'Sullivan \& Gabuzda 2009) reveal Fara- 
day rotations greater than $45^{\circ}$ and a linear dependence of the polarization angle with the wavelength squared. This implies that most of Faraday rotation occurs within the thermal magnetized media located in close proximity to the jet (e.g., Zavala \& Taylor 2004). The detection of a Rotation Measure (RM) gradient across the jet (Asada et al. 2002) in the quasar 3C $273(\mathrm{z}=0.158$; Strauss et al. 1992), and subsequently in other AGN (e.g., Gabuzda et al. 2014; Kravchenko et al. 2017), support the scenario that the sheath is threaded by a helical magnetic field.

A number of studies have revealed that AGN jets change their direction on parsec-scales over time (e.g., Savolainen et al. 2006; Lister et al. 2013), including 3C 273 (Fig. 1). As different components are ejected in different directions into the jet, they fill in the full jet cross-section. Therefore, the true jet width appears wider after stacking together observations performed over many years (Pushkarev et al. 2017; Kovalev et al. 2020). Hereafter we call the latter a wide jet, while a single-epoch jet is called a narrow jet.

3C $273(1226+023)$ has been extensively studied with polarimetric VLBI observations, and it remains the bestestablished case of a transverse RM gradient in AGN jets. In this paper, we present a new multi-epoch highresolution polarimetric study of 3C 273, and Faraday rotation measure analysis, focusing on its long-term evolution, in order to find a connection between the apparent jet orientation and the values of the Rotation Measure. In Section 2, we describe our observations and data reduction steps. In Section 3, we collect several transverse slice measurements from published research and compare them with our measurements. In Section 4, we present a model of an oversized sheath wrapping around the parsec-scale jet in 3C 273 and discuss the geometry of the jet and physical parameters of the plasma in the sheath.

One milliarcsecond corresponds to a projected linear scale of $2.71 \mathrm{pc}$ at the redshift $z=0.158$ of $3 \mathrm{C} 273$, assuming $H_{0}=71 \mathrm{~km} \mathrm{~s}^{-1} \mathrm{Mpc}^{-1}, \Omega_{\mathrm{m}}=0.27$, and $\Omega_{\Lambda}=0.73$. For our calculations we use the viewing angle $\theta=6^{\circ}$ and the corresponding intrinsic full opening angle of the narrow single-epoch jet of $3 \mathrm{C} 273 \alpha_{\text {int }}=1^{\circ}$ (Lisakov et al. 2017).

\section{OBSERVATIONS AND DATA REDUCTION}

The observations were performed with the 10-element Very Long Baseline Array (VLBA, project codes S2087A, B, C, E) on 2009 August 28, 2009 October 25, 2009 December 5, and 2010 January 26 at seven frequencies: $4.6,5.0,8.1,8.4,15.4,23.8,43.2 \mathrm{GHz}$ with a bandwidth of $16 \mathrm{MHz}$ for frequencies below $15.4 \mathrm{GHz}$

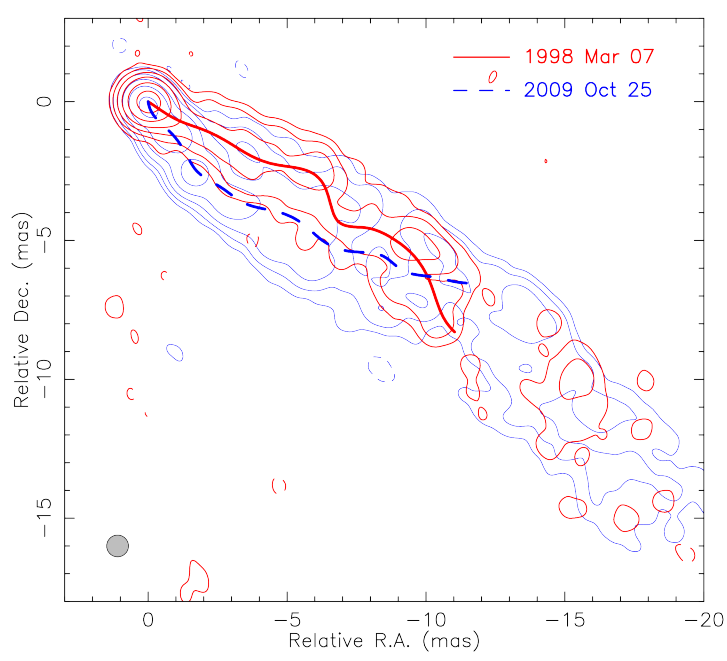

Figure 1. $15 \mathrm{GHz}$ total intensity contours of $3 \mathrm{C} 273$ for epochs 1998 Mar 07 (red, taken from the MOJAVE database) and 2009 Oct 25 (blue, from present study). Both images were restored with the same, average equal-area circular Gaussian FWHM beam size of 0.8 mas (shown in the bottomleft corner) for easier comparison. The images are aligned at the position of the Stokes $I$ peaks. Solid and dashed lines mark the fitted jet ridge lines at the two epochs.

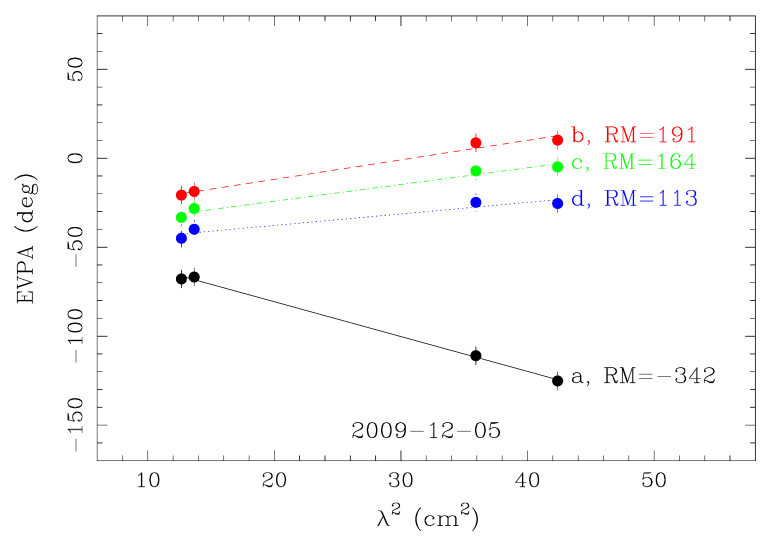

Figure 2. The plot of EVPA vs. $\lambda^{2}$ at locations in the jet shown in Figure 3, with the linear fits and the resultant RM values given in $\mathrm{rad} \mathrm{m}^{-2}$. Colours correspond to those used in Fig. 3.

and $32 \mathrm{MHz}$ for $15.4 \mathrm{GHz}$ and above. For the aims of this study, we consider only the $4.6-15.4 \mathrm{GHz}$ frequency range, while detailed polarization analysis of the quasar in the full frequency range will be presented in a separate publication (Lisakov et al. in prep.). Data reduction and calibration, as well as other techniques used for the analysis were discussed by Lisakov et al. (2017).

The polarization calibration was performed in the same manner as described in Kravchenko et al. (2017) and Kravchenko \& Kovalev (2017), including instrumental polarization of the antennas that was determined with the task LPCAL in AIPS using $1308+326$ as a 
calibrator. An absolute orientation of the electric vector position angle (EVPA) was determined using the net polarization angle of $3 \mathrm{C} 273,3 \mathrm{C} 279$, OJ 287 , and $1308+326$ provided by different monitoring programs ${ }^{1}$ with estimated uncertainty of $5^{\circ}$ and $4^{\circ}$ at $4.6-8.4$ and $15.4 \mathrm{GHz}$, respectively.

Frequency-dependent map offsets were determined using 2D cross-correlation on optically thin portions of the jet (Lisakov et al. 2017) and were used to align images at the different frequencies. Average map offsets in the jet direction are 0.8 mas between 4.6 and $8.1 \mathrm{GHz}$ and 0.6 mas between 8.1 and $15.4 \mathrm{GHz}$. The Faraday rotation analysis was performed separately in two frequency ranges: low $(4.6-8.4 \mathrm{GHz})$ and middle $(8.1-15.4 \mathrm{GHz})$ in order to avoid smearing, which results from a convolution with larger beam sizes. Images at different frequencies were tapered to approximate the resolution of the lower frequency in the corresponding range. All maps were convolved with the restoring beam averaged over four observations at 4.6 and $8.1 \mathrm{GHz}$ in corresponding frequency ranges. We calculated the polarization errors according to Hovatta et al. (2012a), and blanked all pixels whose polarized flux density did not exceed three times the polarization error. The RM is defined as a linear slope of the $\operatorname{EVPA}\left(\lambda^{2}\right)$ dependence. Examples of $\operatorname{EVPA}\left(\lambda^{2}\right)$ fits for different parts of the jet are given in Fig. 2. The solution of the $\mathrm{n} \pi$-ambiguity problem and goodness of the $\lambda^{2}$-fit were determined by minimizing the reduced $\chi^{2}$. Accordingly, the pixels that had $\chi^{2}>5.99$ (which in our case of four data points and two degrees of freedom corresponds to the 95 per cent confidence level) were blanked.

\section{RM IMAGES AND SLICES}

Faraday Rotation Measure images are presented in Fig. 3 for the observations at $4.6-8.4 \mathrm{GHz}$ and in Fig. 4 for the $8.1-15.4 \mathrm{GHz}$ range. Only a single-epoch image is presented, since the RM distribution throughout the jet is consistent over four epochs and does not change dramatically in regions downstream from the core. By the 'core' or 'apparent core', we mean the most northeastern region of the jet in the VLBI images. We associate it with the apparent base of the jet and a surface with an optical depth $\tau \approx 1$ (Blandford \& Königl 1979; Lobanov 1998). The overall RM distribution is smooth and shows persistent transverse gradients throughout

${ }^{1}$ VLBA Polarization Calibration Resources http://www.vla.nrao. edu/astro/calib/polar/, UMRAO database https://dept.astro. lsa.umich.edu/datasets/umrao.php, and MOJAVE database http://www.physics.purdue.edu/astro/MOJAVE/. the whole jet length, at least up to $57 \mathrm{pc}$ in projection along the jet.

The absence of detected Rotation Measure close to the apparent core of $3 \mathrm{C} 273$ is usually attributed to depolarization that happens in this region (e.g. Asada et al. 2002; Hovatta et al. 2012b). We note that during our observations in 2009-2010 there was a major $\gamma$-ray flare detected in 3C 273 and a subsequent flare at radiowavelengths (Lisakov et al. 2017). This is also supported by RadioAstron observations performed in 2012-2014 (Kovalev et al. 2016; Bruni et al. 2017) that show severe variability in the finest-scale structure of $3 \mathrm{C} 273$ which is associated with the apparent core region. This variability together with the low level of polarized emission lead to blanking of the RM values close to the apparent jet base according to our criteria described in Section 2 .

To study the long-term evolution of the Rotation Measure and its gradient across the jet, we have collected previously published results on 3C 273. Asada et al. (2002) and Asada et al. (2008) reported a gradient of $\mathrm{RM}$ values taken across the jet at $4.6-8.6 \mathrm{GHz} 9$ mas downstream from the apparent core. In their observations performed on 1995 December 9, 1995 November 22, and 2002 December 15, RM values across the jet from south to north have ranges $[130,370] \mathrm{rad} \mathrm{m}^{-2}$ and $[170,480] \mathrm{rad} \mathrm{m}^{-2}$, which correspond to a gradient of $\approx 50 \mathrm{rad} \mathrm{m}^{-2} \mathrm{mas}^{-1}$. Zavala \& Taylor (2005) reported a much larger transverse gradient of $500 \mathrm{rad} \mathrm{m}^{-2}$ mas $^{-1}$ taken slightly closer to the apparent core at $12-22 \mathrm{GHz}$. Their observations were performed in 2000 January 27 and 2000 August 11, and also exhibit mostly positive $\mathrm{RM}$ values along the slice, $[150,2350] \mathrm{rad} \mathrm{m}^{-2}$ and $[-50,1900] \mathrm{rad} \mathrm{m}^{-2}$ at the two epochs, respectively.

Wardle (2018) compiled most of the RM measurements including those of Chen (2005) performed in 1999-2000 at $8-22 \mathrm{GHz}$. All RM values transverse to the $3 \mathrm{C} 273$ jet are positive, ranging within $[200,800] \mathrm{rad} \mathrm{m}^{-2}$ at the same location as in the previous works. RM gradients in these observations were $\approx 200 \mathrm{rad} \mathrm{m}^{-2} \mathrm{mas}^{-1}$.

A notable new result came from the research of Hovatta et al. (2012a) based on $8-15 \mathrm{GHz}$ observations on 2006 March 9 and 2006 June 15, where the authors for the first time observed significant negative RM values, in contrast to earlier observations. Hovatta et al. (2012a) report RM values across the jet ranging within $[-600,500] \mathrm{rad} \mathrm{m}^{-2}$, yielding a RM gradient of the same sign $\approx 180 \mathrm{rad} \mathrm{m}^{-2} \mathrm{mas}^{-1}$.

To compare with previous studies, we have analyzed transverse slices from our data taken at 9 mas (as in Asada et al. 2008) and 16 mas (control slice) from the apparent jet base at 4.6-8.4 GHz and 5.5 mas (as in 

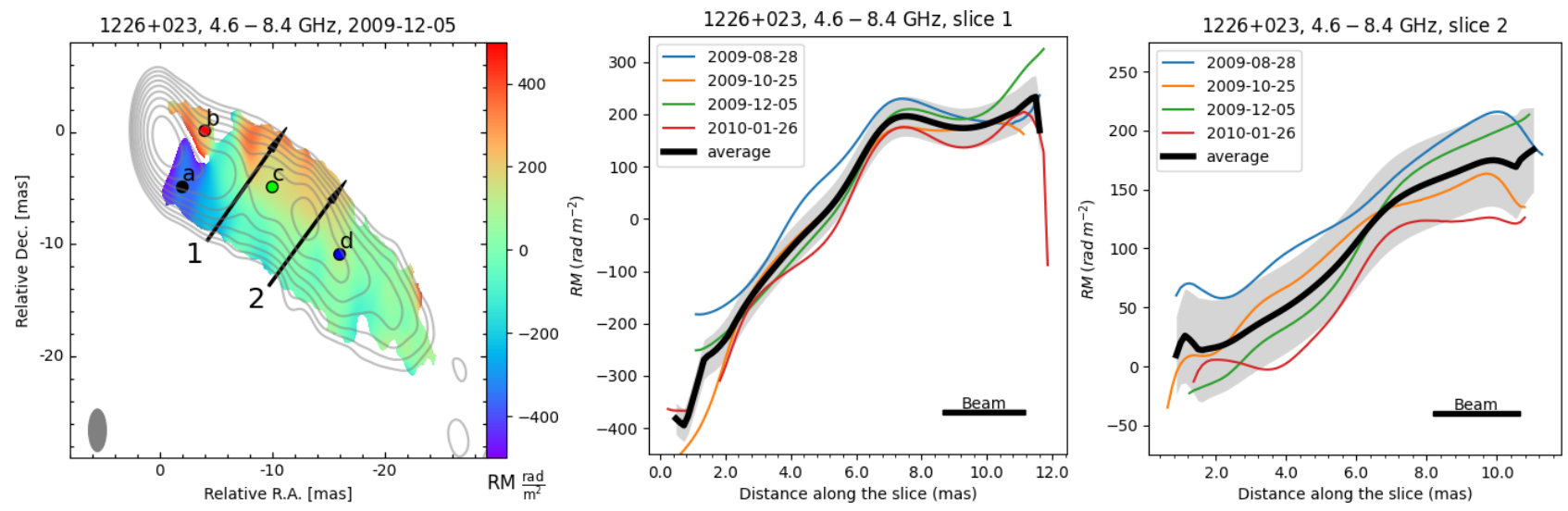

Figure 3. Left: $4.6-8.4 \mathrm{GHz}$ Rotation Measure image (color) overlaid on the $4.6 \mathrm{GHz}$ total intensity contours for $3 \mathrm{C} 273$ at epoch 2009 December 05. The FWHM beam size is indicated in the bottom left corner. Points indicate locations where the $\operatorname{EVPA}\left(\lambda^{2}\right)$ data were extracted as shown in Fig. 2. The location of the slices are displayed by arrows. The first slice (center) is taken at a distance 9 mas from the image center, as in Asada et al. (2008). Right: a reference slice taken at 16 mas from the image center in the region that should not be affected by the jet direction change by 2009. In both slices, color lines represent measured RM values at each of the individual observations, while the thick black line is the average. The shaded area shows the average RM uncertainty. The beam projection FWHM on each slice is shown as a bar.
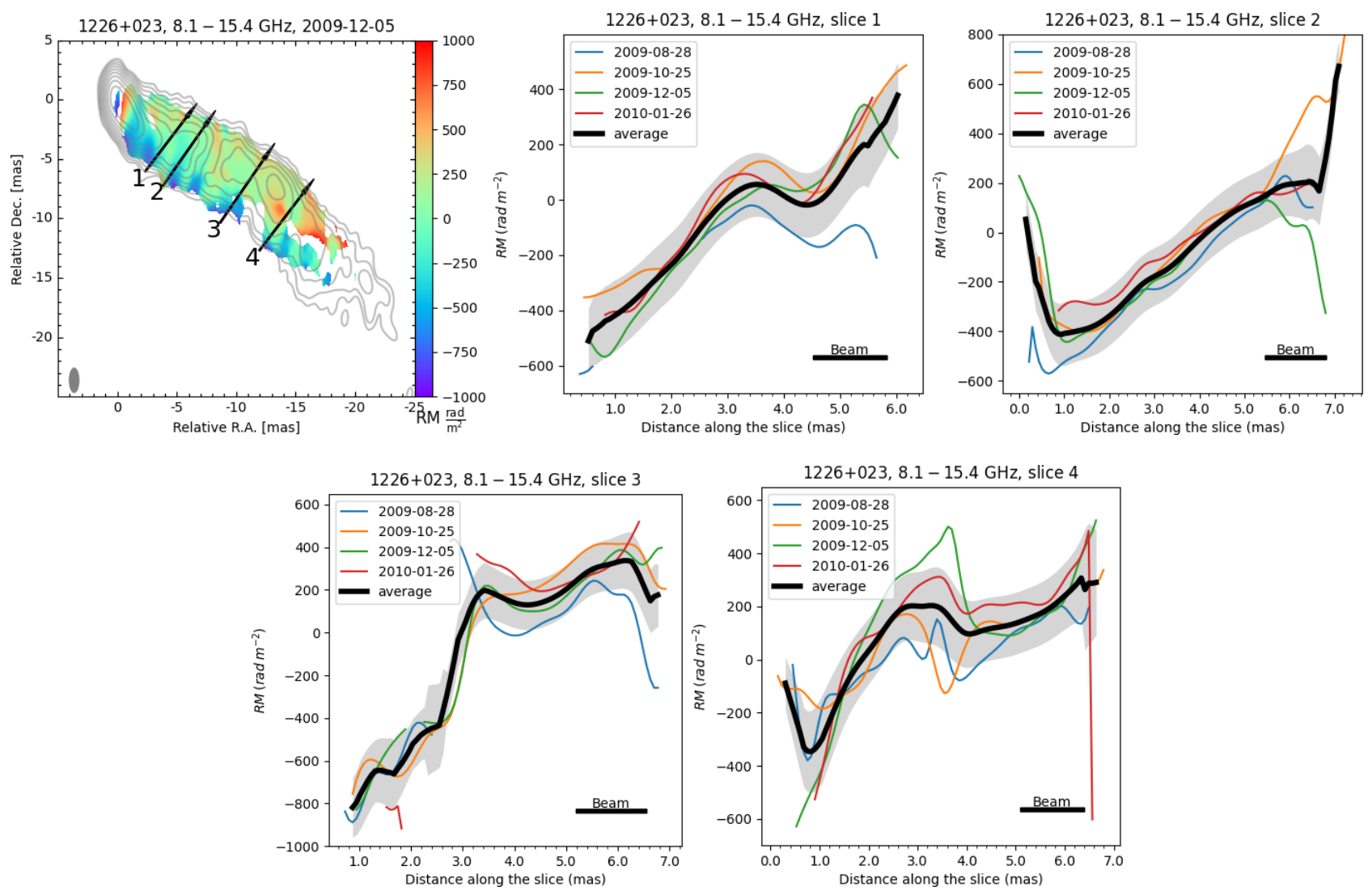

Figure 4. The same as in Fig. 3, but for the 8.1-15.4 GHz range, with the total contours from 8.1 GHz at epoch 2009 Dec 05. 
Zavala \& Taylor 2005), 7 mas (as in Hovatta et al. 2012a, slice 2), 13 mas (as in Hovatta et al. 2012a, slice 3), and 16 mas (control slice) at $8.1-15.4 \mathrm{GHz}$.

As is seen in Fig. 3 (slice 1) and Fig. 4 (slice 1), closer to the apparent core, RM values across the jet are negative at the southern edge of the jet and positive at the northern edge. The range of values is $[-400,200] \mathrm{rad} \mathrm{m}^{-2}$ at $4.6-8.4 \mathrm{GHz}$ and $[-500,400] \mathrm{rad} \mathrm{m}^{-2}$ at $8.1-15.4 \mathrm{GHz}$. The latter agrees well with the measurements of Hovatta et al. (2012a). On the other hand, at 4.6-8.4 GHz, farther from the jet beginning, the RM values are positive throughout the whole cross-section of the jet, see Fig. 3 (slice 2). These positive $\mathrm{RM}$ profiles are more similar to the $4.6-8.6 \mathrm{GHz}$ results of Asada et al. (2008).

At middle frequencies, transverse RM variations are present at every separation from the core, showing negative values at the southern edge and positive at the northern edge. We note, that due to our higher bandwidth and longer integration times we detect a broader $\mathrm{RM}$ distribution across the jet with respect to previous studies, hence more negative RM values are detected at the southern edge of the jet.

\section{DISCUSSION}

\subsection{RM variability}

With a large set of observations that span over 14 years and probe variability timescales down to several months, we can study both long-term and short-term evolution of the Rotation Measure values in the jet. We focus here on comparing transverse slices.

Fig. 3 and Fig. 4 clearly show that within the 5 months covered by our observations, the RM values across the jet at different distances from the apparent core do not change significantly. For the whole jet, net RM values change by no more than by $40 \mathrm{rad} \mathrm{m}^{-2}$ at $4.6-8.4 \mathrm{GHz}$ and by no more than $120 \mathrm{radm}^{-2}$ at $8.1-15.4 \mathrm{GHz}$. These values do not exceed the uncertainty associated with the absolute EVPA calibration. Within the 5month period covered by our observations in 2009-2010 the RM pattern in the jet of 3C 273 has remained largely constant, consistent with the measurements of Zavala \& Taylor (2005) over 6 months and three years. This is in contrast to the short timescale variability over three months as reported by Hovatta et al. (2012a).

When considering variability on timescales of years, there are notable changes in the RM distribution in the jet of 3C 273. Asada et al. (2008) reported on the small changes in the RM over 7 years. But a major change appears to have occurred some time between 2003 and 2006. Namely, at 7 mas downstream the jet, there are now negative values at the southern edge of the jet.
This was first discovered by Hovatta et al. (2012a) and is fully confirmed with our observations at both 4.68.4 GHz and 8.1-15.4 GHz, see e.g. Fig. 3 (slice 1) and Fig. 4 (slice 1).

Although there are alternative explanations of the long-term evolution of the RM values, we argue that anything outside the immediate jet vicinity fails to explain variability on the timescale of several years and large changes in RM, considering the high Galactic latitude $b=64.4^{\circ}$ of $3 \mathrm{C} 273$ (Taylor et al. 2009).

\subsection{Geometry of the jet of 3C273}

According to Lister et al. (2013), the narrow jet of 3C 273 had abruptly changed its apparent direction by $20^{\circ}$ in 2003. Fig. 1 shows these structural variations in the quasar jet in application to the observations of 1998 March 7 and 2009 October 25 at 15 GHz. Assuming a constant viewing angle of the overall quasar jet axis of $\theta=6^{\circ}$ (Lisakov et al. 2017), this translates into an intrinsic jet direction change of $2^{\circ}$, larger than the intrinsic opening angle measured at a single epoch $\alpha_{\text {int }}=1.1^{\circ}$ (Lisakov et al. 2017).

With a median apparent jet speed of 0.8 mas $\mathrm{yr}^{-1}$ (Lister et al. 2019), structural changes should affect the jet by 2010 within about 7 mas from its apparent beginning, consistent with Fig. 1. We also note that some moving features in the jet of $3 \mathrm{C} 273$ have larger apparent speeds, up to 1.5 mas $\mathrm{yr}^{-1}$, which could potentially double the affected jet region (Savolainen et al. 2006).

\subsection{Wide Faraday sheath in 3 C 273}

We suggest that the Faraday rotating medium consists of an oversized sheath wrapped around the wide jet instead of being tightly connected with the narrow, singleepoch jet as shown in Fig. 5. The inner opening angle of the sheath is therefore larger than the opening angle of the narrow single-epoch jet that wiggles within the inner opening angle of the oversized sheath. In this scenario, there is a misalignment between the visible narrow relativistic jet axis and the symmetry axis of a broader conical sheath which is threaded with toroidal magnetic field. Therefore, due to the swing in the parsec-scale jet direction occurring in 2003, synchrotron radiation passed through different regions of the Faraday screen and different RM values were observed before and after this change. This possibility was previously considered to explain some RM changes in 3C 273 (Hovatta et al. 2012b) and 3C 120 (Gómez et al. 2011).

There are several possible ways to verify this scenario. Firstly, the screen is detached from the relativistic jet and most probably has a much slower velocity. Therefore, it should evolve relatively slowly. Indeed, for our data, we have measured the jet net RM 


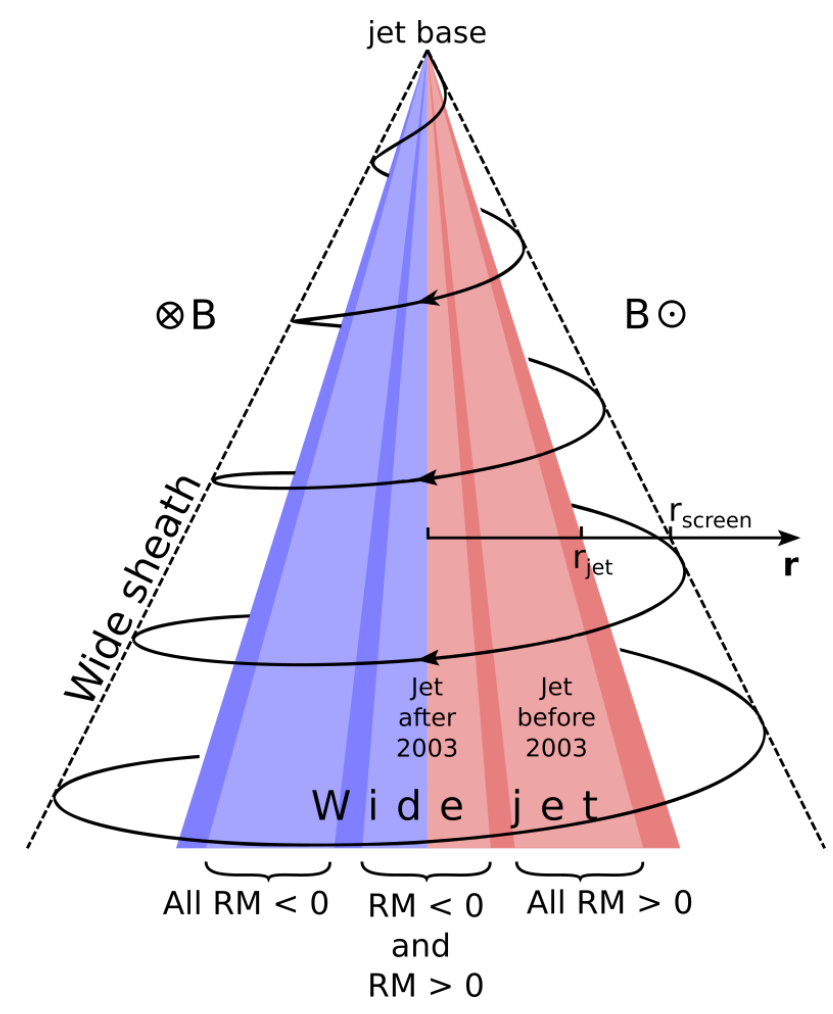

Figure 5. Sketch of a sheath wrapped around the wide jet. Subject to the single-epoch relativistic jet orientation, synchrotron emission of the jet passes through different parts of the sheath. RM values are all positive before 2003 (rightmost jet position) and cross zero after 2003 (middle jet position). If the jet of $3 \mathrm{C} 273$ turns more to the south (to the leftmost position in this sketch), only negative $\mathrm{RM}$ values will be observed.

changes between observations in 2009-2010 as: $-32,-3$, $-25 \mathrm{rad} \mathrm{m}^{-2}$ at $4.6-8.4 \mathrm{GHz}$ and $114,-34,63 \mathrm{rad} \mathrm{m}^{-2}$ at $8.1-15.4 \mathrm{GHz}$. These values are within the uncertainties associated with the EVPA calibration. Secondly, should the rotating medium constitute a sheath around the narrow relativistic jet with thickness comparable to the jet cross-section, it will inevitably be destroyed by the relativistic jet once it changes direction. However, we do not detect any traces of such an interaction.

We note that Park et al. (2019) present RM images of the jet in the radio galaxy M87 within the Bondi radius and conclude that the dominant source of the observed significantly high RMs are hot winds. The latter are non-relativistic, moderately magnetized gas outflows that surround the highly magnetized jet of M87 and are threaded by toroidally-dominated magnetic fields. But M87 shows no RM gradients across its jet. Park et al. (2019) suggest that this is due to a misalignment between the jet axis and the symmetry axis of the toroidal field loops in the Faraday screen, therefore the RM gradients are not observed in M87 since only a portion of the sheath is illuminated. Although the matter which forms the sheath in M87 and in 3C 273 may have a different origin, observational properties for both sources are well explained within the presented model.

Our model of an oversized sheath explains the longterm RM variability in the inner parts of the jet with different apparent directions of the relativistic jet before and after 2003 and hence the jet synchrotron emission passing through different parts of the Faraday screen. We can also predict that if the jet direction changes farther to the south, mostly negative RM values will be observed.

\subsection{Screen parameters}

To estimate parameters of the Faraday screen we have developed a simple model. The wide jet with a radius $r_{\text {jet }}$ is wrapped by a hollow conical sheath with inner radius $r_{\text {jet }}$ and outer radius $r_{\text {screen }}$, see Fig. 5 . The toroidal component of the magnetic field in the sheath decays with perpendicular distance from the wide jet axis as $B_{\|}=B_{1} r^{-1}$ and the thermal particle density declines as $N_{\mathrm{e}}=N_{1} r^{-2}$ where $B_{1}$ and $N_{1}$ are taken at 1 pc from the wide jet axis. After integrating $R M \propto \int B_{\|} N_{\mathrm{e}} d l$ over different lines of sight perpendicular to the apparent jet direction, we obtained the distribution of the RM values across the jet width. We then convolved these with a Gaussian restoring beam assuming five beams across the jet to resemble the data at 4.6-8.4 GHz. This simple model is designed to account only for smooth and monotonic distribution of RM across the jet and give a hint of typical parameters of the medium in the real sheath.

For any $r_{\text {screen }}$, the inner layers of the screen, up to $r_{\text {screen }}=3 \times r_{\text {jet }}$, contribute $75 \%$ of the observed RM as expected from the model, see Fig. 6 . This supports our initial assumption that the sheath would be disrupted by the jet changing its direction if this sheath is located in the immediate vicinity of the narrow relativistic jet. Moreover, the predicted RM distribution across the jet is well described by a straight line.

One may assume that the maximum value of the RM across the jet at 9 mas from the apparent core measured by Asada et al. (2002) was $500 \mathrm{rad} \mathrm{m}^{-2}$ and that the minimum value, not observed yet, would be $-500 \mathrm{rad} \mathrm{m}^{-2}$. Then with our measurements of $\mathrm{RM}$ values across the jet at $4.6-8.4 \mathrm{GHz}$ taken at the same distance, we estimate a total wide jet intrinsic opening angle of $\alpha_{\text {int }}=2.1^{\circ}$, twice as large as single-epoch estimates (Lisakov et al. 2017). To perform this, we combine transverse RM distributions, taken at different epochs, with the narrow jet position angle changes taken into account, as is shown in Fig. 7. The apparent angular width of the RM distribution at a single epoch is estimated as 


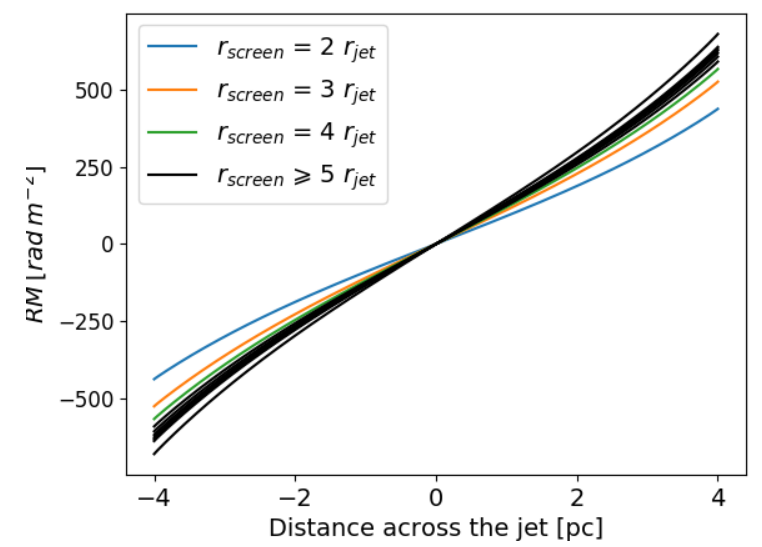

Figure 6. Model RM values across the jet for the 4.6$8.4 \mathrm{GHz}$ range at 9 mas from the core. $B_{1}=12 \mu G$ and $N_{1}=2000 \mathrm{~cm}^{-3}$ were chosen to obtain the total range $[-500,500] \mathrm{rad} \mathrm{m}^{-2}$ of observed $\mathrm{RM}$ values for $r_{\text {screen }}=$ $3 r_{\text {jet }}$. The family of black curves represents $r_{\text {screen }}$ values ranging from $5 r_{\text {jet }}$ to $100 r_{\text {jet }}$.

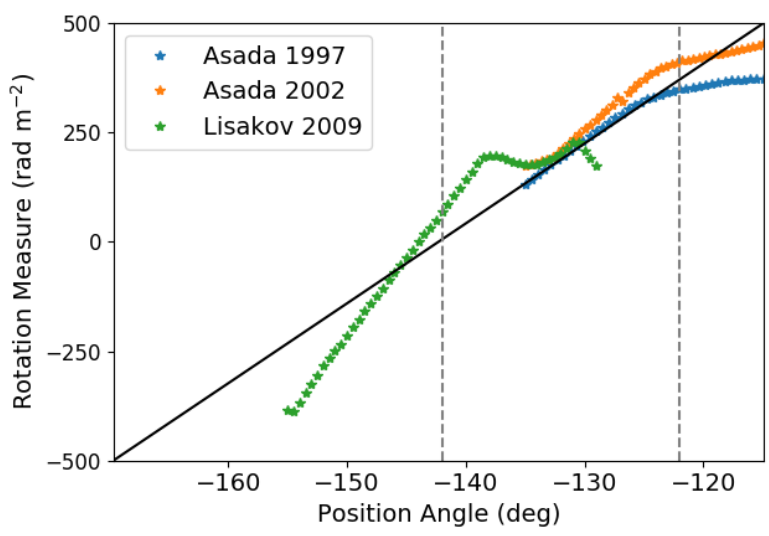

Figure 7. The RM values at $4.6-8.4 \mathrm{GHz}$ taken at 9 mas from the apparent jet beginning transverse to its direction. Blue and orange points represent two epochs, 1997 and 2002, reported by Asada et al. (2008), while green points are taken from our observation on Dec 05, 2009. The two vertical dashed lines show the average narrow jet position angle before $\left(\right.$ at $-122^{\circ}$ ) the major jet swing in 2003, and after (at $-142^{\circ}$ ). The black diagonal line represent a linear fit to the combined data set.

the width of the transverse jet intensity profile taken at the $1 \%$ level. Afterwards, the combined transverse RM distribution was fitted with a linear function, in accordance with the model. The total range of jet position angles, required to obtain a $[-500,500] \mathrm{rad} \mathrm{m}^{-2}$ range of the RM, is $55^{\circ}$ which corresponds to the intrinsic opening angle of the wide jet $\alpha_{\text {int }}=2.1^{\circ}$.

With this estimate, at 9 mas from the apparent core, the total width of the wide jet is $8 \mathrm{pc}, r_{\text {jet }}=4 \mathrm{pc}$. As we found for an arbitrary large $r_{\text {screen }}$, the inner layers of the sheath up to $r=3 r_{\text {jet }}$ are responsible for $75 \%$ of the rotation. Hence we assumed $r_{\text {screen }}=3 r_{\text {jet }}$. The range of $\mathrm{RM}$ values $[-500,500]$ then requires $B_{1} \times N_{1}=$ $24000 \mu \mathrm{G} \mathrm{cm}^{-3}$ for a path length $2 r_{\text {jet }}=8 \mathrm{pc}$ through the sheath. Clearly, $B_{1}$ and $N_{1}$ can not be calculated independently within this analysis. At the same time, the line-of-sight path length through the screen could not change the estimates of $B_{1} \times N_{1}$ by more than a factor of two within our model, for any reasonable screen width. With $r_{\text {jet }}=4 \mathrm{pc}, B_{\|} \times N_{\mathrm{e}}=375 \mu \mathrm{Gcm}^{-3}$ at the wide jet - wide sheath boundary. For assumed magnetic field component parallel to the line of sight $B_{\|}=3 \mu \mathrm{G}$ at the inner edge of the sheath, the thermal particle density is $N_{\mathrm{e}}=125 \mathrm{~cm}^{-3}$.

With the same logic applied to the $8.1-15.4 \mathrm{GHz}$, a range of $\mathrm{RM}$ values $[-2000,2000]$ could be achieved at 5.5 mas from the jet beginning with $B_{1} \times N_{1}=$ $37500 \mu G \mathrm{~cm}^{-3}$, as obtained from the model with path length of $2 r_{\text {jet }}=5$ pc. $B_{1} \times N_{1}$ is $25 \%$ higher than that at 9 mas, what is expected given the lower distance to the jet beginning. As is apparent from Fig. 4, transverse RM distributions observed at higher frequencies in general show more complex shape and thus require a more elaborate model to describe them. This is a scope of future work that will allow a better estimate of the Faraday screen parameters.

It is important to note that using the core shift analysis (Lobanov 1998) and estimates of the magnetic field $B_{0}=0.47 \mathrm{G}$ and relativistic electron density $N_{0}=$ $1500 \mathrm{~cm}^{-3}$ at the jet beginning (Lisakov et al. 2017), the estimated values in the jet are $B_{140}=3 \mu \mathrm{G}, N_{140}=$ $8 \times 10^{-2} \mathrm{~cm}^{-3}$ at 5.5 mas (corresponding to $140 \mathrm{pc}$, deprojected) and $B_{230}=2 \mu \mathrm{G}, N_{230}=3 \times 10^{-2} \mathrm{~cm}^{-3}$ at $9 \operatorname{mas}(230 \mathrm{pc}$, deprojected) from the apparent jet base.

\subsection{The jet opening angle derived from stacked images}

We produced a stacked map of $3 \mathrm{C} 273$ in total intensity at $15 \mathrm{GHz}$ (Fig. 8) using public data from the MOJAVE database (Lister et al. 2019). According to discussion in Section 4.3, this map represents the wide jet in 3C 273. In total, 105 epochs ranging from 1995 July 28 to 2019 April 15 were stacked together. Each single-epoch image was convolved with the same median circular beam and shifted such that the $15 \mathrm{GHz}$ VLBI core is placed in the phase center. The core position (Lister et al. 2019) was determined from source structure modeling performed in the Fourier plane with the procedure modelfit from the Caltech DiFMAP package (Shepherd 1997). We fitted a ridge line to the image and measured the apparent wide jet opening angle $\alpha_{\text {app }}$ following the procedure described in Pushkarev et al. 


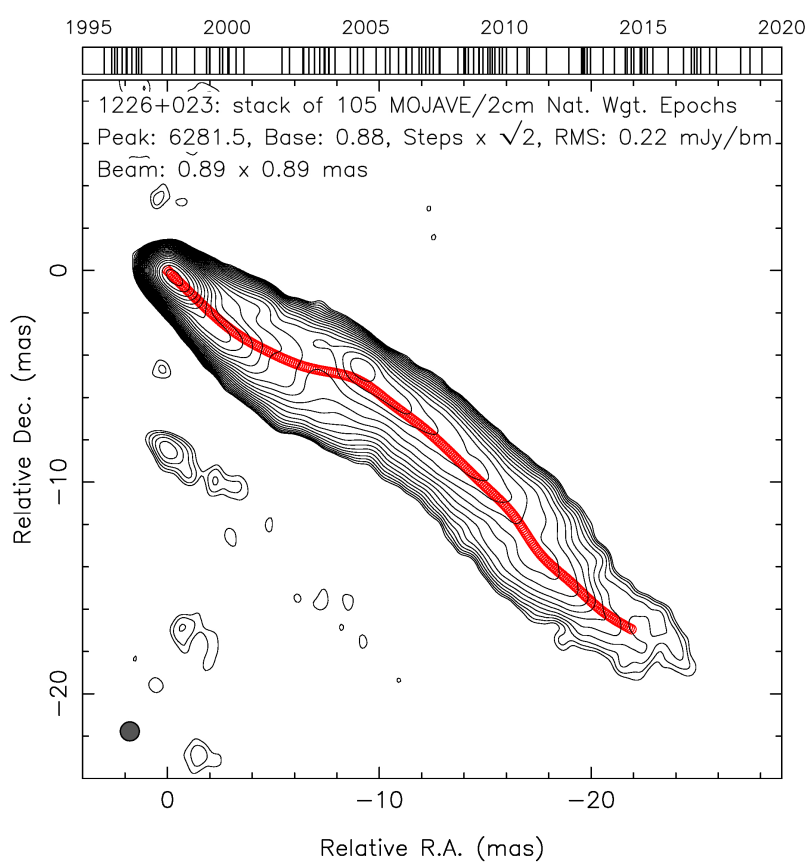

Figure 8. $15 \mathrm{GHz}$ naturally weighted stacked CLEAN image of 3C 273 based on 105 VLBA epochs from the MOJAVE program (Lister et al. 2019). The contours are shown at increasing powers of $\sqrt{2}$ times the base contour level of 0.88 mJy beam ${ }^{-1}$. The restoring beam dimensions are plotted as a shaded circle in the lower left corner. The constructed total intensity ridge line is depicted in red. A wedge indicating observing epochs (vertical ticks) used for producing the stacked image is shown on top.

(2017). The intrinsic wide jet opening angle, calculated as $\tan \left(\alpha_{\text {int }} / 2\right)=\tan \left(\alpha_{\text {app }} / 2\right) \sin \theta$, is shown in Fig. 9. The intrinsic opening angle of the wide jet is $\alpha_{\text {int }} \approx 2^{\circ}$ up to the distances of about 9 mas from the core along the ridge line, in noticeable agreement with the estimate made above under the assumption on the minimum Rotation Measure $-500 \mathrm{rad} \mathrm{m}^{-2}$.

We also note that high resolution space VLBI images of 3C 273 obtained with RadioAstron at both $1.6 \mathrm{GHz}$ and $4.8 \mathrm{GHz}$ and presented recently by Bruni et al. (2021) show a clear evidence for the new established direction of the jet in $3 \mathrm{C} 273$.

\section{SUMMARY}

We present new high-resolution images of the Faraday Rotation Measure in the parsec-scale jet of 3C 273, see Section 3, based on multifrequency VLBA polarimetric observations at 4.6-15.4 GHz. From the comparison of our results with the previous studies, we confirm the existence of transverse RM gradients in the jet of $3 \mathrm{C} 273$ out to at least $57 \mathrm{pc}$ projected distance (500 pc deprojected), and detect a change of RM towards negative values compared with observations performed before 2003 .

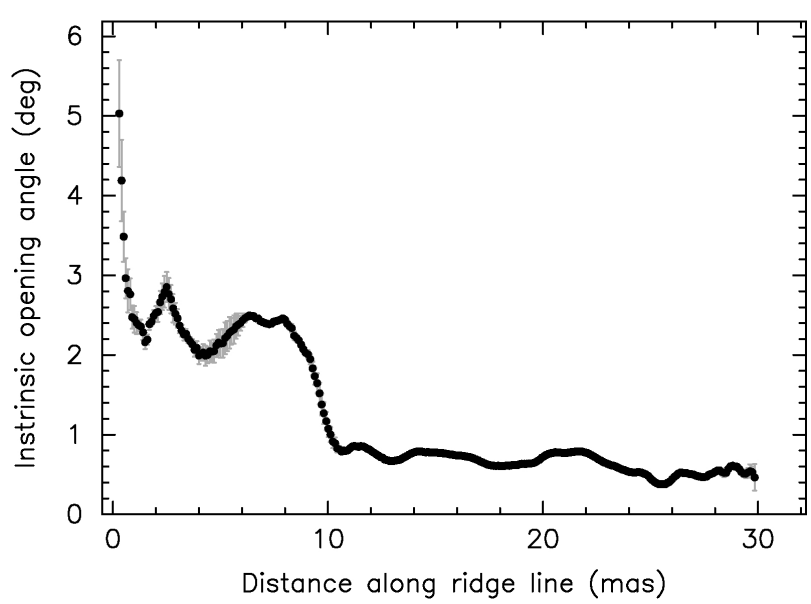

Figure 9. Intrinsic opening angle of the jet derived from stacked image in Fig. 8 as a function of the core separation measured along the ridge line.

In order to coherently explain the change of the jet position angle together with the change of the Faraday Rotation Measure across the jet, in Section 4.3 we develop a model of an oversized sheath that is wrapped around the wide jet in $3 \mathrm{C} 273$. The sheath does not show any rapid variability and most likely is disconnected from the relativistic jet. Single-epoch images of 3C 273 reveal only a portion of the wider jet. After the parsec-scale jet direction swing in 2003, different lines-of-sight through the sheath are sampled, resulting in different observed Faraday Rotation Measure values. We predict that mostly negative RM values will be observed if the jet turns even more southward. Within our model we estimate the total wide jet intrinsic opening angle $\alpha_{\text {int }}=2.1^{\circ}$, based solely on the RM measurements across the jet. Most of the rotation occurs within a layer of $3 \times r_{\text {jet }}$ width, see Section 4.4. In this scenario, we estimate $B=3 \mu \mathrm{G}$ and $N=125 \mathrm{~cm}^{-} 3$ at the jet-sheath border at 9 mas (230 pc, deprojected) from the apparent core at $5 \mathrm{GHz}$.

We produced a stacked image of 3C 273 in total intensity at $15 \mathrm{GHz}$ using VLBA data at 105 epochs of observations performed during 24 years mainly within the MOJAVE program and its predecessor, the $2 \mathrm{~cm}$ VLBA survey, see Section 4.5. The intrinsic wide jet opening angle derived from the transverse cuts is about $5^{\circ}$ at the jet region near the $15 \mathrm{GHz}$ core. The outflow quickly collimates to $\alpha_{\text {int }} \approx 2^{\circ}$. This regime holds within distances from about 1 to 9 mas from the core, further supporting our model. 


\section{ACKNOWLEDGMENTS}

The authors thank the anonymous referee and Nicholas MacDonald for thoroughly reading the manuscript and providing useful comments. We thank Elena Nokhrina for a fruitful discussion while preparing this manuscript. This study has been supported in part by the Russian Science Foundation project 20-7210078. TS was partly supported by Academy of Finland projects 274477 and 315721. This research has made use of data from the MOJAVE database that is maintained by the MOJAVE team (Lister et al. 2018). The MOJAVE program is supported under NASA-Fermi grant 80NSSC19K1579. This research has made of use of VLBA, which is run by the National Radio Astronomy Observatory is a facility of the National Science Foundation operated under cooperative agreement by Associated Universities, Inc. This work made use of the Swinburne University of Technology software correlator (Deller et al. 2011), developed as part of the Australian Major National Research Facilities Programme and operated under licence.

\section{Facilities: VLBA}

Software: AIPS (van Moorsel et al. 1996), Difmap (Shepherd 1997), astropy (Astropy Collaboration et al. 2013), WebPlotDigitizer https://automeris. io/WebPlotDigitizer

\section{REFERENCES}

Asada, K., Inoue, M., Kameno, S., \& Nagai, H. 2008, ApJ, 675, 79, doi: 10.1086/524000

Asada, K., Inoue, M., Uchida, Y., et al. 2002, PASJ, 54, L39, doi: 10.1093/pasj/54.3.L39

Astropy Collaboration, Robitaille, T. P., Tollerud, E. J., et al. 2013, A\&A, 558, A33, doi: 10.1051/0004-6361/201322068

Blandford, R. D., \& Königl, A. 1979, ApJ, 232, 34, doi: $10.1086 / 157262$

Bruni, G., Gómez, J. L., Casadio, C., et al. 2017, A\&A, 604, A111, doi: 10.1051/0004-6361/201731220

Bruni, G., Gómez, J. L., Vega-García, L., et al. 2021, arXiv:2101.07324 [astro-ph]. http://arxiv.org/abs/2101.07324

Chen, T. 2005, PhD thesis, Brandeis University, Massachusetts, USA

Deller, A. T., Brisken, W. F., Phillips, C. J., et al. 2011, PASP, 123, 275, doi: 10.1086/658907

Gabuzda, D. C., Reichstein, A. R., \& O'Neill, E. L. 2014, MNRAS, 444, 172, doi: 10.1093/mnras/stu1381
Gómez, J. L., Roca-Sogorb, M., Agudo, I., Marscher, A. P., \& Jorstad, S. G. 2011, ApJ, 733, 11, doi: 10.1088/0004-637X/733/1/11

Hovatta, T., Lister, M. L., Aller, M. F., et al. 2012a, AJ, 144, 105, doi: 10.1088/0004-6256/144/4/105

Hovatta, T., Lister, M. L., Aller, M. F., et al. 2012b, in Journal of Physics Conference Series, Vol. 355, Journal of Physics Conference Series, 012008, doi: 10.1088/1742-6596/355/1/012008

Kovalev, Y. Y., Pushkarev, A. B., Nokhrina, E. E., et al. 2020, MNRAS, 495, 3576, doi: 10.1093/mnras/staa1121

Kovalev, Y. Y., Kardashev, N. S., Kellermann, K. I., et al. 2016, ApJL, 820, L9, doi: 10.3847/2041-8205/820/1/L9

Kravchenko, E., \& Kovalev, Y. 2017, Galaxies, 5, 92, doi: 10.3390/galaxies5040092

Kravchenko, E. V., Kovalev, Y. Y., \& Sokolovsky, K. V. 2017, MNRAS, 467, 83, doi: 10.1093/mnras/stx021

Lisakov, M. M., Kovalev, Y. Y., Savolainen, T., Hovatta, T., \& Kutkin, A. M. 2017, MNRAS, 468, 4478, doi: $10.1093 / \mathrm{mnras} / \mathrm{stx} 710$ 
Lister, M. L., Aller, M. F., Aller, H. D., et al. 2018, ApJS, 234, 12, doi: 10.3847/1538-4365/aa9c44

—. 2013, AJ, 146, 120, doi: 10.1088/0004-6256/146/5/120

Lister, M. L., Homan, D. C., Hovatta, T., et al. 2019, ApJ, 874, 43, doi: 10.3847/1538-4357/ab08ee

Lobanov, A. P. 1998, A\&A, 330, 79. https://arxiv.org/abs/astro-ph/9712132

McKinney, J. C. 2006, MNRAS, 368, 1561, doi: 10.1111/j.1365-2966.2006.10256.x

Meier, D. L., Koide, S., \& Uchida, Y. 2001, Science, 291, 84, doi: 10.1126/science.291.5501.84

Nakamura, M., Asada, K., Hada, K., et al. 2018, ApJ, 868, 146, doi: 10.3847/1538-4357/aaeb2d

O'Sullivan, S. P., \& Gabuzda, D. C. 2009, MNRAS, 393, 429, doi: 10.1111/j.1365-2966.2008.14213.x

Park, J., Hada, K., Kino, M., et al. 2019, ApJ, 871, 257, doi: 10.3847/1538-4357/aaf9a9

Pushkarev, A. B., Kovalev, Y. Y., Lister, M. L., \& Savolainen, T. 2017, MNRAS, 468, 4992, doi: 10.1093/mnras/stx854

Savolainen, T., Wiik, K., Valtaoja, E., \& Tornikoski, M. 2006, A\&A, 446, 71, doi: 10.1051/0004-6361:20053753

Shepherd, M. C. 1997, in Astronomical Society of the Pacific Conference Series, Vol. 125, Astronomical Data Analysis Software and Systems VI, ed. G. Hunt \& H. E. Payne (San Francisco: ASP), 77
Strauss, M. A., Huchra, J. P., Davis, M., et al. 1992, ApJS, 83, 29, doi: 10.1086/191730

Taylor, A. R., Stil, J. M., \& Sunstrum, C. 2009, ApJ, 702, 1230, doi: 10.1088/0004-637X/702/2/1230

Tchekhovskoy, A., Narayan, R., \& McKinney, J. C. 2011, MNRAS, 418, L79, doi: 10.1111/j.1745-3933.2011.01147.x

van Moorsel, G., Kemball, A., \& Greisen, E. 1996, in Astronomical Society of the Pacific Conference Series, Vol. 101, Astronomical Data Analysis Software and Systems V, ed. G. H. Jacoby \& J. Barnes, 37

Vlahakis, N., \& Königl, A. 2004, ApJ, 605, 656, doi: $10.1086 / 382670$

Wardle, J. 2018, Galaxies, 6, 5, doi: 10.3390/galaxies6010005

Zamaninasab, M., Clausen-Brown, E., Savolainen, T., \& Tchekhovskoy, A. 2014, Nature, 510, 126, doi: 10.1038/nature13399

Zavala, R. T., \& Taylor, G. B. 2003, ApJ, 589, 126, doi: $10.1086 / 374619$

—. 2004, ApJ, 612, 749, doi: 10.1086/422741

—. 2005, ApJL, 626, L73, doi: 10.1086/431901 